\title{
Orgulho e agradecimentos
}

\author{
Harley E. A . Bicas, Cristina Muccioli, Mauro Campos, Mauro Goldchmit, Samir J. Bechara, Vital Paulino Costa
}

Ao analisar 231 títulos de periódicos científicos brasileiros, definindo-os quanto a critérios de alcance ("nacional" ou "local") e classificando-os pela qualidade (níveis "A", "B", "C" ou "sem classificação"), o CNPq, por meio de um seleto grupo de assessores, enquadrou os Arquivos Brasileiros de Oftalmologia com os melhores conceitos de abrangência (um dos 156 reconhecidos como "nacionais") e, dentre estes, de qualidade (um dos 51 com o mérito do critério "A"). Fomos portanto reconhecidos como entre os $22 \%$ das revistas científicas nacionais de maior difusão e expressão. Já não seria pouco.

Por outro lado, em uma pesquisa independente, recentemente divulgada (outubro de 1999), um sextanista de Medicina da E.P.M - U.N.I.F.E.S.P. (Cláudio Csillag) elaborou, com a base de dados da Bireme (Centro Latino-Americano e do Caribe de Informação em Ciências da Saúde), uma metodologia para a classificação de revistas científicas, estabelecendo a adequação formal e editorial de 182 publicações, cadastradas pela LILACS (Literatura-Latino Americana e do Caribe em Ciências da Saúde), em 1997. O estudo considerava critérios de avaliação assumidos pelos mais expressivos indexadores internacionais de dados na área da Saúde (Index Medicus, LILACS, Excerpta Medica, Institute for Scientific Information). Em uma escala de zero a cem, 109/182 = 59,9\% das publicações receberam notas de 60 a 90 . Mas importante é que na lista das vinte revistas que ficaram com a maior pontuação (portanto apenas $11 \%$ do universo analisado), estava o nome dos Arquivos Brasileiros de Oftalmologia. Isso deve ser motivo de muito orgulho para todos os oftalmologistas brasileiros e vem premiar o esforço dos que lutam para tornar nossos A.B.O. cada vez melhores.

E por falar em esforços e contribuições cabe, ao fim de mais um ano de trabalhos, tornar públicos os agradecimentos editoriais àqueles que até então de forma anônima e generosa analisaram matérias que nos foram encaminhadas, opinando sobre suas apresentações e conteúdos, sugerindo mudanças, corrigindo, auxiliando autores e advertindo-os sobre omissões ou impropriedades de seus artigos: os revisores.

Uma publicação não sobrevive sem autores. Mas são os revisores que lhe garantem a qualidade final, separando o joio do trigo, propondo ajustes, refinando, protegendo reputações. Progressivamente, começam os autores a perceber as vantagens de uma revisão de seus trabalhos, tão extensa e crítica quanto possível. Uma publicação, como o próprio nome diz, torna públicos os acertos e erros de um autor, expondo-o aos quatro ventos e perenemente. Infeliz o autor sem a vanta- gem de um conselho (para evitar, por exemplo, o aparecimento constrangedor de deficiências), despreparado aquele que apenas vê intromissões do revisor, sem lhe reconhecer, pelo menos, o mérito da dúvida levantada que, certamente, assaltaria também outros leitores. O revisor é um "anjo da guarda" do trabalho, um seu leitor atento (e só isso deveria torná-lo simpático ao autor). Claro que nem sempre o revisor está certo e, pois, nem sempre é o autor obrigado a acatá-lo. Mas, felizmente, já são muitos os autores que manifestam agradecimentos a esses "coautores" anônimos que, na maior parte das vezes, estão mesmo com a razão e contribuem com suas sugestões para o aperfeiçoamento da apresentação.

É a esse grupo de gente, trabalhando ética e sigilosamente nos bastidores de cada publicação, que queremos agora agradecer. Não só às pessoas que apareceram relacionadas como membros de nosso Conselho Redatorial mas também às que, além delas, foram acionadas "ad hoc", para revisões. Em nosso nome, mas principalmente nos dos autores a quem anonimamente serviram; e igualmente nos dos leitores, cujos benefícios recebidos de uma leitura mais amena, mais consistente, mais correta, nem sequer são às vezes pressentidos, os agradecimentos todos a:

Adriana Berezovisky
Amélia Kamegasawa
Ana Cláudia Alves Pereira
Ana Tereza Ramos Moreira
André Luís Borba da Silva
Antonio Augusto Velasco e Cruz
Antonio Marcelo B. Casella
Augusto Paranhos Jr.
Belquiz R. do Amaral Nassaralla
Carlos Akira Omi
Carlos Rubens Lucas de Figueiredo
Cecilia Lapa
César Lipener
Christiane Regina Rolim de Moura
Cláudio Lisias N. Macedo
Clélia Maria Erwenne
Cristina Maria Bittencourt Garrido
Denise de Vuono Chinzon
Denise Fornazari de Oliveira
Diane Ruschel Marinho
Edison João Geraissate Filho


Edméa R. Temporini Nastari

Eduardo Jorge Carneiro Soares

Eduardo Martines

Eduardo Melani Rocha

Eduardo Pinheiro Penna

Eduardo Sone Soriano

Eliana Aparecida Forno Velasco

Elisabeto Ribeiro Gonçalves

Ernesto Consoni Filho

Felício Aristóteles da Silva

Fernando Kayat Avvad

Flávio Jaime da Rocha

Francisco Eduardo Lopes de Lima

Hamilton Moreira

Hélia Soares Angotti

Ítalo Mundialino Marcon

João Antonio Prata Junior

João Francisco Cêntola Nóbrega

José Américo Bonatti

José Antonio de Almeida Milani

José Augusto Cardillo

José Eduardo L. Dolci

José Paulo Cabral Vasconcellos

José Wilson Cursino

Joyce Hisae Yamamoto

Juliana Maria Ferraz Sallum

Keila Míriam M. de Carvalho

Lúcia Mélega Ré

Luciene Barbosa de Souza

Luís Carlos Ferreira de Sá

Luiz Alberto Molina Mônica

Luiz Antonio Vieira

Magno Antonio Ferreira

Manuel Augusto Pereira Vilela

Márcia Beatriz Tartarella

Márcia Motono

Marco Cezar Helena

Marcos Wilson Sampaio

Marcus Vinicius Abbud Safady

Maria Auxiliadora M. F. Sibinelli

Maria Carmen Menezes Santos

Maria Cristina Nishiwaki Dantas
Maria de Lourdes M. M. Villas Boas

Maria Kiyoko Oyamada

Maria Rosa Bet de M. Silva

Maria Teresa Brizzi Chizzotti Bonanomi

Marilisa Nano Costa

Mário Junqueira Nóbrega

Marisa Braga Poterio

Marta Beatriz C. F. Sartori

Maurício Maia

Mírian Skaf

Mônica Fialho Cronemberger

Nilo Holzchuh

Odair Guimarães

Paulo Afonso Batista dos Santos

Paulo Elias Correa Dantas

Paulo Góis Manso

Paulo Schor

Procópio Miguel dos Santos

Ralph Cohen

Regiani Lopes Malicia Bauzys

Ricardo Themudo Lessa Waetge

Ricardo Uras

Roberli H. Bicharra Pinto

Roberto Caldato

Roberto Freire Santiago Malta

Rosane da Cruz Ferreira

S. Eloy Pereira

Saly Maria Bugmann Moreira

Seiji Hayashi

Sidney Júlio de F. e Sousa

Sílvia Veitzman

Solange Rios Salomão

Sonia Regina Almeida A. Pinheiro

Suzana Matayoshi

Tomás F. Scalamandré Mendonça

Vera Christina Waller de Lima

Vera Regina C. Castanheira

Vicente Muniz de Carvalho

Virgílio A. M. D. Centurión

Walter Yukihiko Takahashi

Wesley Ribeiro Campos

\section{Novidades na Internet:?!}

Agora no site CBO você tem disponível todas as informações na íntegra dos

Arquivos Brasileiros de Oftalmologia

http://www.cho.com.br/abo 